\title{
ASSIMILAÇÃO DO IWV-GPS NO BRASIL: OTIMIZAÇÃO DAS ESTIMATIVAS DO ATRASO ZENITAL TROPOSFÉRICO EM TEMPO REAL
}

\author{
Luiz Fernando Sapucci ${ }^{1}$, João Francisco Galera Monico², Luiz Augusto Toledo Machado , \\ Dirceu Luis Herdies ${ }^{1}$ e Rodrigo Augusto Ferreira de Souza ${ }^{1}$
}

Recebido em 30 abril, 2007 / Aceito em 12 julho, 2007

Received on April 30, 2007 / Accepted on July 12, 2007

\begin{abstract}
IWV (Integrated Water Vapor) values from the Brazilian Network for Continuous Monitoring (RBMC) of GPS (Global Positioning System) signals are additional sources of humidity information available for being used in Numerical Weather Prediction (NWP) models operating in the Brazilian meteorological centers. In order to obtain IWV-GPS values in an efficient approach, an optimization of the GPS data processing for estimating the ZTD values (Zenithal Tropospheric Delay) is involved, which should search for the best result with lowest computational cost. In this optimization process it is necessary to determine the ideal size of the so called sliding-window data involved in the real time data processing, which must be the as small as possible but providing results with the required quality. In this paper is investigated the ideal size of such sliding-window. An experiment was carried out in which GPS sliding-windows of different sizes were evaluated using simulations of a real time data processing. Comparing the obtained results with those from post-processed GPS data, it is observed that a sliding-window containing 7 hours of GPS data generated the best results from the operational point of view (rms of 1.61 and $1.82 \mathrm{~kg} \mathrm{~m}^{-2}$ for latency of 60 and 120 minutes, respectively).
\end{abstract}

Keywords: IWV-GPS, ZTD, NWP, data assimilation.

RESUMO. Valores do IWV (Integrated Water Vapor - Vapor D’água Integrado na coluna atmosférica) provenientes da Rede Brasileira de Monitoramento Contínuo (RBMC) dos Satélites GPS (Global Positioning System - Sistema de Posicionamento Global) compõem uma fonte adicional de informações da umidade para a assimilação em modelos operacionais de Previsão Numérica de Tempo (PNT) existentes no Brasil. Para que os valores do IWV-GPS sejam obtidos com maior eficiência é necessário uma otimização do processamento de dados GPS para a estimativa do ZTD (Zenithal Tropospheric Delay - Atraso Zenital Troposférico), de forma que resultados com qualidade adequada possam ser obtidos com custo computacional baixo. Nessa otimização deve-se definir o tamanho ideal da chamada janela deslizante (sliding window) de dados GPS envolvidos no processamento em tempo real, a qual deve ser a menor possível, mas fornecendo resultados satisfatórios. 0 objetivo desse trabalho é investigar sobre o tamanho ideal dessa janela de dados. Um experimento foi realizado envolvendo janelas de dados GPS de diferentes tamanhos, as quais foram testadas através de simulações de processamento em tempo real. Comparando os resultados simulados com aqueles obtidos no modo pós-processado, observou-se que do ponto de vista operacional o emprego de uma janela com 7 horas de dados proporciona os melhores resultados (Erro médio Quadrático de 1,61 e $1,82 \mathrm{~kg} \mathrm{~m}^{-2}$ para 60 e 120 minutos de latência, respectivamente).

Palavras-chave: IWV-GPS, ZTD, PNT, assimilação de dados.

\footnotetext{
${ }^{1}$ Centro de Previsão de Tempo e Estudos Climáticos (CPTEC), Instituto Nacional de Pesquisas Espaciais (INPE), Rodovia Presidente Dutra, km 40 - 12630-000 Cachoeira Paulista, SP, Brasil. Tel.: (12) 3186-9400 - E-mails: Isapucci@cptec.inpe.br / machado@cptec.inpe.br / dirceu@cptec.inpe.br / rodrigo@cptec.inpe.br 2Programa de Pós Graduação em Ciências Cartográficas (PPGCC), Universidade Estadual Paulista (UNESP), Rua Roberto Simonsen, 305 - 19060-900 Presidente Prudente, SP, Brasil. Tel.: (18) 3229-5352 - E-mail: galera@fct.unesp.br
} 


\section{INTRODUÇÃ̃O}

0 emprego das redes terrestres de monitoramento contínuo dos sinais GPS (Global Positioning System - Sistema de Posicionamento Global) na quantificação do IWV (Integrated Water Vapor - Vapor D'água Integrado na coluna atmosférica) (daqui para frente denominado IWV-GPS) é uma aplicação multidiciplinar com grande potencial para o monitoramento da concentração do vapor d'água atmosférico, a qual apresenta boas perspectivas para diversos estudos relacionados às ciências atmosféricas. Diversos projetos têm explorado essa técnica envolvendo centros de pesquisas na área de Meteorologia e de Geodésia em vários países. Como exemplos, pode-se destacar o SUOMINET ( Rea/Time National GPS Network for Atmospheric Research and Education) (Ware et al., 2000), nos Estados Unidos da América; 0 GASP (GPS Atmosphere Sounding Project), que é responsável pela aplicação da densa rede de receptores GPS da Alemanha na PNT (Reigber et al., 2001); o COST-716 (European Co-operation in the Field of Scientific and Technical Research 716), que envolve instituiçõos de 15 diferentes países europeus (Marel, 2000); e 0 "GPS/MET Japan" (Tsuda et al., 1998), com uma rede de quase 1000 estações GPS criada inicialmente para monitoramento de abalos sísmicos. Seguindo essa tendência mundial, esforços têm sido realizados para possibilitar a utilização da Rede Brasileira de Monitoramento Contínuo dos Satélites GPS (RBMC) (Fortes, 1997) no monitoramento do IWV sobre o território Brasileiro. 0 objetivo é disponibilizar mais uma fonte de informações sobre 0 vapor d'água atmosférico para os modelos de Previsão Numérica de Tempo (PNT). A inclusão do IWV apresenta um benefício em potencial para a PNT que é minimizar os erros na estrutura vertical da umidade com impactos positivos para a previsão de fenômenos atmosféricos, principalmente para a previsibilidade da precipitação pluviométrica a curto prazo (Kuo et al., 1996).

0 principio básico da PNT consiste em obter o estado futuro da atmosfera em uma instante $t$ a partir do conhecimento de seu estado inicial em um instante $t_{0}$. Para tanto, emprega-se as leis de evolução desse estado. 0 sucesso na previsão está relacionado à capacidade de se modelar, com maior eficiência, a atmosfera terrestre e, principalmente, descrever com precisão o seu estado inicial. Para isso, o maior número possível de dados sobre as variáveis atmosféricas deve ser disponibilizado no momento em que o processo é iniciado. Nisso está envolvido o que é conhecido por "latência" dos dados, que pode ser definida como sendo o período compreendido entre o instante em que os mesmos estão disponíveis a um determinado processo e o instante ao qual eles se referem. No caso das informações atmosféricas, os fatores res- ponsáveis pela latência estão relacionados ao tempo gasto nos processos de coleta, armazenamento e disponibilização. Para que a eficiência da PNT seja obtida necessita-se que a maior quantidade possível de dados com a menor latência seja disponível à esse processo.

Para 0 caso dos valores do IWV a partir das observações GPS, além dos fatores responsáveis pela latência já mencionados, existe também o tempo de processamento dos dados para a estimativa de valores do ZTD (Zenithal Tropospheric Delay Atraso Zenital Troposférico) em tempo real (Rocken et al., 1997b), os quais são convertidos em valores do IWV (Bevis et al., 1992). Para que tais valores tenham a menor latência é necessário que 0 método de processamento seja otimizado, de forma que o melhor resultado seja obtido com o menor custo computacional. Apesar do grande avanço computacional dos últimos anos ter tornado 0 custo computacional de muitos processos pouco relevante, em circunstâncias onde está envolvida uma grande quantidade de observações esse fator ainda é crítico e merece ser investigado. Esse é 0 caso dos receptores GPS, pois os mesmos rastreiam simultaneamente pelo menos 8 satélites realizando 7 tipos diferentes de observação a cada 15 segundos, o que totaliza em média 13.000 observações por hora. Para essa otimização devese definir qual é 0 tamanho ideal da chamada janela deslizante (em inglês denominado sliding window) de dados GPS envolvidos no processamento em tempo real, de modo que seja a menor possível e que forneça os melhores resultados. 0 objetivo desse trabalho é investigar qual é 0 tamanho ideal da janela deslizante que otimize as estimativas do IWV em tempo real. Para isso, um experimento foi realizado onde diferentes janelas de dados são testadas através de simulações de processamento em tempo real. A avaliação é feita ao comparar os resultados obtidos com valores pós-processados. Como o processo de utilização de dados atmosféricos por um modelo de PNT, denominado assimilação, está diretamente relacionado ao tema principal desse trabalho, uma breve descrição desse processo é apresentada, destacando os detalhes mais relevantes do ponto de vista da latência dos valores do IWV obtidos a partir das observáveis GPS.

\section{ASSIMILAÇÃO DOS VALORES DO IWV-GPS EM MODELOS DE PNT NO BRASIL}

0 processo de assimilação de dados em um modelo de PNT é um procedimento cíclico onde os resultados gerados pelo modelo de PNT em uma integração curta $(t-6)$, são usados como valores iniciais (denominado FG do inglês first guess) pelo sistema de assimilação. Nesse processo, o FG é combinado com 
as observações disponíveis, em um instante $t$, gerando um estado da atmosfera, denominado de análise, que estatisticamente é considerado 0 estado ótimo que mais se aproxima da realidade física nesse instante (Kalnay, 2003). Essa análise é utilizada para a integração do modelo de PNT como condição inicial para gerar, tanto o FG do próximo passo $(t+6)$, como as previsões do estado da atmosfera válidas para épocas futuras $(t+6, t+12, t+18, \ldots)$. Informações adicionais sobre a assimilação de dados em PNT podem ser obtidas em Herdies et al. (2002), o qual apresenta um esquema ilustrativo dos processos envolvidos.

Atualmente no Brasil, apesar de haver vários centros de previsão que empregam modelos de PNT operacionalmente, 0 CPTEC-INPE (Centro de Previsão de Tempo e Estudos Climáticos do Instituto Nacional de Pesquisas Espaciais) é o único que realiza assimilação de dados em seus modelos de PNT. Os demais centros apenas executam seus modelos a partir da condição inicial fornecida por outros, como o NCEP (National Centers for Environmental Prediction) ou o ECMWF (European Center for MediumRange Weather Forecasts). 0 sistema de assimilação utilizado no CPTEC é o PSAS (Physical-space Statistical Analysis System) (Da Silva \& Guo, 1996; Cohn et al., 1998), desenvolvido no DAO/NASA (Data Assimilation Office/National Aeronautics Space Administration). 0 modelo global de PNT utilizado no CPTEC é o CPTEC-COLA (Kinter et al., 1997; Cavalcanti et al., 2002), com resoluções de $200 \times 200,100 \times 100$ e $63 \times 63 \mathrm{~km}$ (valores próximos ao equador). Para a América do Sul é utilizado o modelo regional ETA (Black, 1994) com resoluções de $40 \times 40$ e $20 \times 20 \mathrm{~km}$ (valores próximos ao equador).

Tanto para o modelo global como para o modelo regional, o PSAS é executado 4 vezes diariamente com análises sendo geradas nos seguintes horários: 00, 06, 12 e 18 GMT. Seguindo a mesma denominação utilizada nas ciências atmosféricas, tais horários serão tratados aqui por sinóticos (resumidos). A Figura 1 apresenta um esquema dos horários que se referem às rodadas do PSAS, destacando os momentos em que ele é acionado e 0 tamanho da janela de dados utilizado. Nessa figura duas configurações são apresentadas: uma atual e uma futura em fase de estudo. Observa-se que atualmente o PSAS é executado 3 horas após os horários sinóticos e que a janela de dados é de \pm 2 horas. Na futura configuração o mesmo será executado 2 horas após os horários sinóticos, utilizando uma janela de \pm 1 hora de dados. Essa configuração futura é para privilegiar as previsões de curto prazo, que serão geradas adicionalmente às obtidas nas atuais rodadas. 0 esquema da Figura 1 mostra que atualmente, para as observações que se referem aos horários sinóticos, a latência máxima final permitida pelo processo de assimilação é de 180 minutos, a qual passará para 120 minutos na futura configuração. Esses valores da latência devem ser levados em consideração ao otimizar a obtenção das estimativas do IWV a partir das observações GPS quase que em tempo real.

\section{Determinação das estimativas do atraso zenital troposférico quase em tempo real}

Há diversos fatores que influenciam as observáveis GPS gerando erros na determinação da posição da antena GPS (Monico, 2000). Entre eles está 0 atraso nos sinais GPS gerado pela influência dos gases que compõem a atmosfera terrestre. Para a quantificação do IWV utilizando o GPS é necessário estimar com precisão oS valores desse atraso na direção zenital. Isso é obtido ao aplicar uma estratégia adequada que minimize os demais erros presentes nas determinações GPS (Sapucci, 2001), além de funções de mapeamento que relacionam o atraso na direção satélite-receptor com o mesmo na direção zenital (Spilker, 1996).

Na estimativa do ZTD para obtenção dos valores do IWVGPS em tempo real o maior problema está relacionado com os erros nas efemérides (posição dos satélites) e nos relógios dos satélites GPS utilizados no processamento (Rocken et al., 1997a). As opções para solucionar esse problema são basicamente duas. Na primeira opção, as posições dos satélites são preditas a partir do processamento dos dados GPS coletados por receptores pertencentes a redes de monitoramento contínuo. Essas efemérides são utilizadas no processamento em tempo real visando obter as estimativas do ZTD, levando em consideração a época em que foram geradas, pois a qualidade de sua predição é degradada no tempo (Rocken et al., 1997a). A segunda opção é através da utilização das efemérides ultra-rápidas disponibilizadas pelos centros de análise do IGS (International GNSS Service), as quais foram criadas especialmente para esse fim (NASA, 2005). Diversos trabalhos têm sido desenvolvidos visando avaliar a qualidade das órbitas ultra-rápidas e os resultados obtidos mostram que elas possuem alta qualidade (Reigber et al., 2001). Tais resultados tornaram a primeira opção citada acima inviável e por esse motivo, no presente estudo, foi utilizada a segunda opção.

Outro problema associado às estimativas do atraso zenital troposférico em tempo real está relacionado às etapas de predição, filtragem e suavização do filtro de Kalman envolvido nesse processo. Essas etapas não são adequadamente executadas em toda a janela de dados utilizada no processo, o que gera um aumento da incerteza dos valores obtidos no início e no final da janela considerada. Enquanto no início esse aumento é devido 
(a) Configuração atual
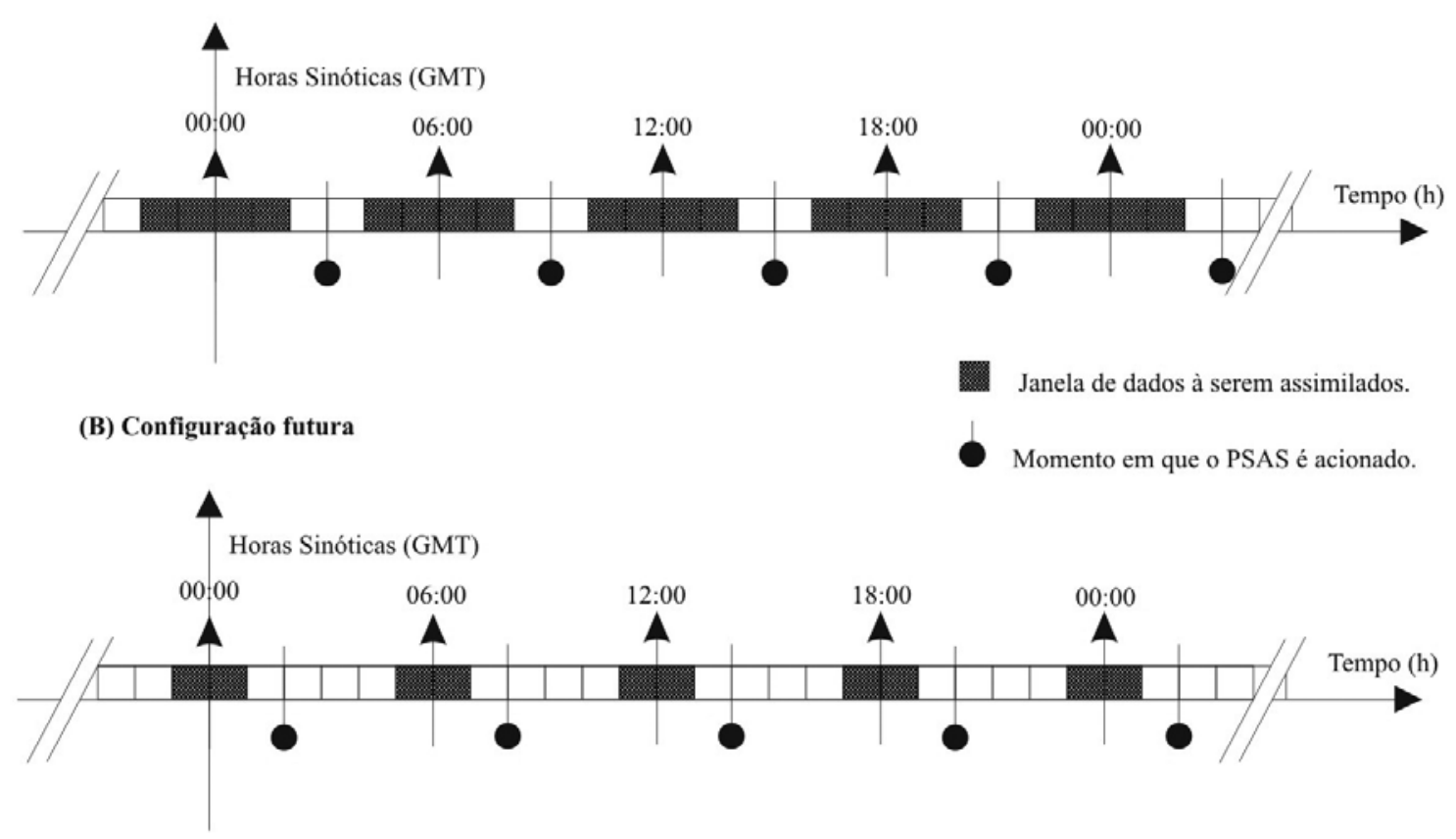

Figura 1 - Esquema ilustrativo das execuções operacionais do PSAS: (a) configuração atual e (b) configuração futura visando previsões a curto prazo no CPTEC.

ao processo de predição, no fim da janela é devido à execução precária do processo de suavização. Esse fato está intimamente ligado à latência das estimativas do atraso zenital troposférico, pois as estimativas mais precisas, em uma determinada época, só estarão disponíveis depois de certo tempo. Esse tempo é necessário para que o processo de suavização seja executado suficientemente nessa época, aumentando a qualidade final das estimativas. Esse intervalo de tempo está associado com o tamanho da janela de dados utilizado no processamento. Assim, os valores do atraso podem ser estimados com uma latência de 1,5 h (Marel, 2000), 1 h (Reigber et al., 2001) e até 30 min ou menos (Rocken et al., 1997a), dependendo do tamanho da janela de dados utilizada e da precisão requerida. A determinação do tamanho da janela ideal é algo que merece atenção (Foster et al., 2005), pois ela está associada com a eficiência do processo e a qualidade final das estimativas do IWV-GPS.

\section{Determinação do IWV a partir das estimativas do atraso zenital troposférico}

Devido ao tipo de comportamento dos gases que compõem a troposfera, que para fins geodésicos pode ser considerada como sendo a camada próxima a superfície com até $50 \mathrm{~km}$ de altura, o ZTD é dividido em duas componentes: a úmida $\left(D_{Z W}-Z e-\right.$ nithal Wet delay), formada pela influência do vapor d'água, e a hidrostática $\left(D_{Z H}-\right.$ Zenithal Hydrostatic delay), formada pela influência dos demais gases que compõem a atmosfera (Spilker, 1996). A componente hidrostática depende apenas da densidade do ar atmosférico e, por isso, se considerado o equilíbrio hidrostático, seus valores podem ser determinados a partir de medidas de pressão à superfície $\left(P_{0}\right)$, da latitude do local $(\varphi)$ e da altitude $(h)$ em quilômetros, segundo a seguinte equação (Davis et al., 1985):

$$
\begin{aligned}
Z_{H D}= & \left(2,27671422 \cdot 10^{-3}\right) \\
& \times\left(P_{0}\right) /(1-0,0026 \cos 2 \varphi-0,00028 h),
\end{aligned}
$$

Assim, ao tomar um valor do atraso zenital troposférico obtido a partir das observações GPS e subtrair o atraso troposférico da componente hidrostática chega-se no valor do atraso zenital da componente úmida $\left(D_{Z W}\right)$. A relação entre os valores do $D_{Z W}$ e a quantidade de vapor d'água atmosférico (IWV) existente no momento em que foram feitas as observações GPS é função da temperatura média troposférica $(T m)$. Essa relação pode ser expressa pela seguinte equação (Bevis et al., 1992):

$$
I W V=D_{Z W} \cdot \frac{10^{6}}{R_{w}\left[k_{2}^{\prime}+\frac{k_{3}}{T m}\right]},
$$


onde $R_{w}=(461,5181) \mathrm{Jkg}^{-1} \mathrm{~K}^{-1}$ é a constante específica para 0 vapor d'água, $k_{2}^{\prime}=22,10 \mathrm{~K} \mathrm{hPa}^{-1}$ e $k_{3}=373900 \mathrm{~K}^{2} \mathrm{hPa}^{-1}$ são as constantes da refratividade atmosférica cujos valores foram determinados experimentalmente (Bevis et al., 1994). Os valores de $T m$ podem ser aproximados a partir de medidas de temperatura efetuadas à superfície (Bevis et al., 1992). Para regiões do território brasileiro uma modelagem adequada que relaciona os valores de $T m$ com dados obtidos à superfície foi desenvolvida empregando-se 90.000 radiossondas (Sapucci et al., 2004).

\section{EXPERIMENTO PARA AVALIAÇÃO DO TAMANHO IDEAL DA JANELA DE DADOS}

Para determinar o tamanho ideal da janela de dados, realizou-se um experimento onde diferentes tamanhos foram testados. Os critérios utilizados para a avaliação dos diferentes casos testados estão relacionados com a otimização do processo, ou seja, melhor qualidade com o menor tempo de processamento (menor latência). Na avaliação da qualidade, os resultados obtidos numa simulação de um processamento em tempo real são comparados com valores obtidos no pós-processamento.

Os dados GPS utilizados nesse experimento são provenientes da estação UEPP da RBMC, localizada em Presidente Prudente - SP (latitude $22^{\circ} 07^{\prime} \mathrm{S}$, longitude $51^{\circ} 24^{\prime} \mathrm{W}$ e $435 \mathrm{~m}$ de altitude). 0 receptor utilizado (marca Trimble, modelo 4000SSI e antena modelo Dorne Margolin, do tipo Choke Ring) foi configurado para disponibilizar as observações GPS em arquivos contendo uma hora de dados, que atualmente é a configuração padrão que tem sido adotada em diversas redes de coleta de dados. 0 período de coleta dos dados utilizados foi de 1/6/2004 a 30/6/2004.

As efemérides utilizadas na simulação do processamento em tempo real foram as do tipo ultra-rápida produzida pelo JPL (Jet Propulsion Laboratory) (Gregorius, 1996). Essas efemérides diferem das fornecidas por outros centros em dois aspectos. Elas não apresentam predição para épocas futuras àquela em que foram disponibilizadas e as coordenadas dos satélites e os erros dos relógios são disponibilizados em um intervalo de 5 minutos. Para o pós-processamento dos dados as efemérides utilizadas foram as do tipo precisa, também produzidas pelo JPL.

0 software utilizado para o processamento dos dados GPS, tanto em tempo real como pós-processados, foi o GOA-II (Gregorius, 1996), adotando máscara de elevação de $10^{\circ}$, intervalo de processamento de 30 segundos e a função de mapeamento de Niell (Niell, 1996), sendo que o processo estocástico para estimar o ZTD foi o Random Walkcom o valor do sigma do processo estocástico de $5 \mathrm{~mm} \mathrm{~h}^{-1 / 2}$. Para tratar dos erros gerados pela influência da ionosfera na propagação do sinal GPS foi utilizado a combinação linear Ion-Free, a qual é apropriada para minimizar tais efeitos (Klobuchar, 1996).

A metodologia utilizada nesse experimento consiste basicamente no processamento de um conjunto de dados de diferentes tamanhos, utilizando efemérides ultra-rápidas e comparando com os resultados obtidos com o processamento convencional pós-processado usando efemérides precisas e arquivos de dados de 24 horas. Esse procedimento pode ser dividido nas seguintes etapas:

1. As diferentes janelas de dados avaliadas foram formadas ao concatenar arquivos no formato RINEX contendo observações GPS coletadas num período de uma hora. Para simular uma janela deslizante no tempo, cada um desses arquivos foi posicionado no final de um dos arquivos concatenados. Dessa forma, foram gerados 24 arquivos concatenados em cada um dos dias do experimento;

2. Para simular o processamento em tempo real, as efemérides ultra-rápidas obtidas via ftp (sideshow.nasa.gov.br) foram organizadas de forma que, para o processamento de cada um dos arquivos obtidos na etapa 1 , elas contivessem as mesmas características da versão obtida em tempo real;

3. Para as diferentes janelas de dados consideradas foi feito um processamento utilizando as efemérides ultra-rápidas do JPL. Foram processados todos os 30 dias considerados na campanha, gerando um total de 720 processamentos para cada uma das janelas testadas. A Figura 2 apresenta um esquema ilustrativo dos 720 processamentos para 0 caso da janela de 6 horas de dados. 0 intervalo de processamento dos dados foi de 30 segundos, como mencionado anteriormente, e as estimativas do ZTD foram geradas em um intervalo de 5 minutos;

4. Com o objetivo de se isolar todas as influências externas aos resultados obtidos com as duas formas de processamento dos dados, os valores de temperatura e pressão atmosférica utilizados na conversão dos valores do ZTD em IWV foram constantes. Por esse motivo, para o cálculo do $Z_{H D}$ a Eq. (1) foi substituída pela seguinte equação (Blewitt, 1997):

$$
\begin{aligned}
Z_{H D} \cong & 1,013 \times 2,27 \\
& \times \exp \left(-h \times 0,116 \times 10^{-3}\right)
\end{aligned}
$$




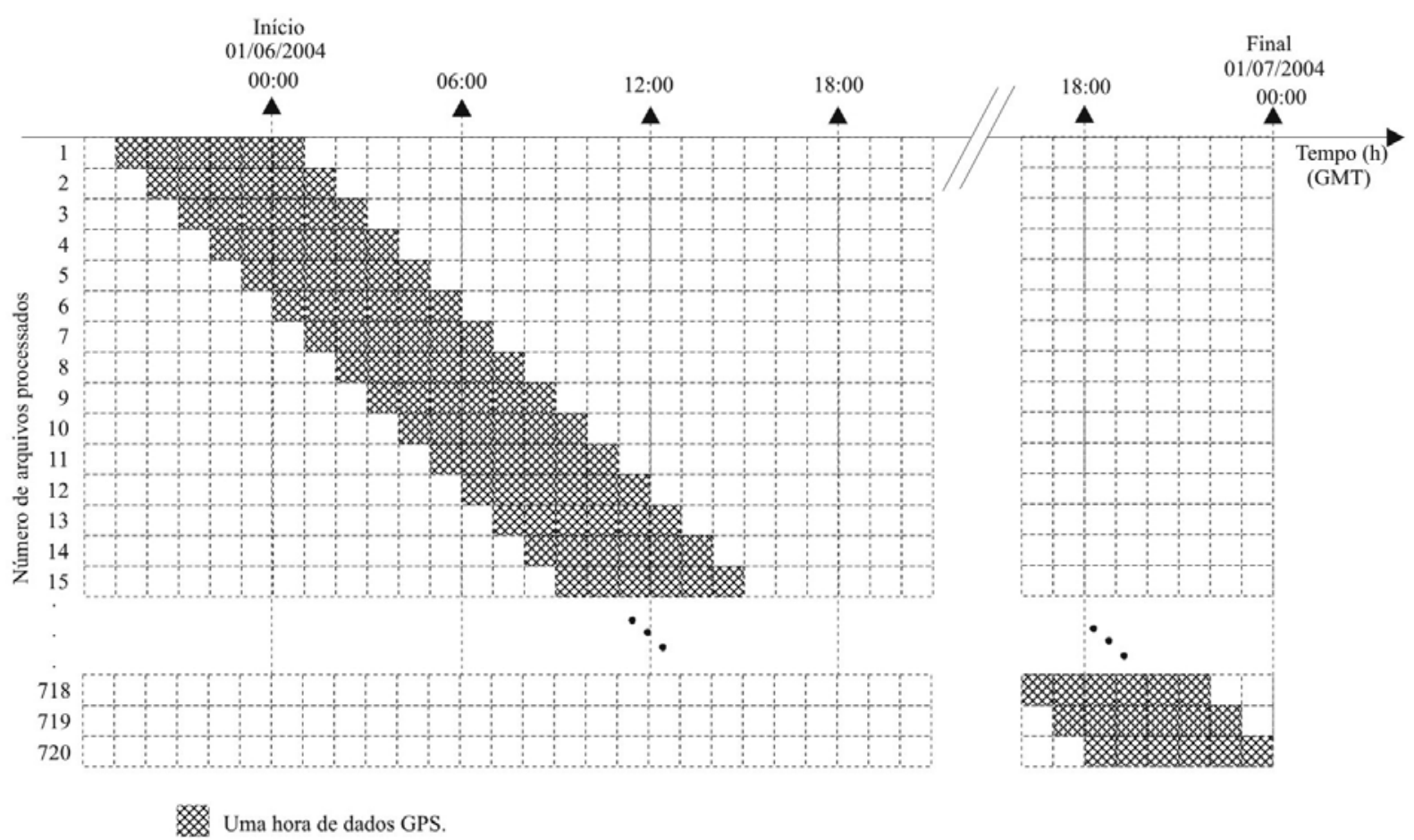

Figura 2 - Esquema ilustrativo da janela de 6 horas de dados deslizante no tempo, dentro do período considerado nesse estudo.

na qual os valores do $Z_{H D}$ são função apenas de valores da altitude $h$ da estação. Para a estação UEPP o valor de $h$ aplicado na Eq. (3) foi de 430,95 m, o que resultou em um valor constante do $Z_{H D}$ de 2,187 m. Para a aplicação da Eq. (2), o valor da $T m$ utilizado foi 0 sugerido por Sapucci (2001) e válido para essa estação, que é de $276,38 \mathrm{~K}$.

Os tamanhos das janelas de dados testados foram selecionados de forma interativa, com escolha de janelas nos extremos e na metade do intervalo de 24 horas em conjunto com análises preliminar dos resultados obtidos. Inicialmente foram selecionadas janelas de 1 hora (mínimo), 24 horas (máximo) e 12 horas (metade). Uma análise preliminar dos resultados obtidos com tais janelas indicou que o intervalo entre 1 e 12 horas deveria ser avaliado. Esse intervalo foi dividido em 3 partes, selecionando assim janelas de 3, 6 e 9 horas de dados. Uma segunda análise indicou a necessidade de se avaliar melhor as janelas próximas de 6 horas, sendo assim selecionadas também janelas de 5 e 7 horas.

\section{RESULTADOS}

Para a avaliação dos resultados obtidos com as diferentes janelas de dados foram calculados o viés (medida de tendência) e o EMQ
(Erro Médio Quadrático) (medida de acurácia), considerando os valores pós-processados como referência nessa análise de qualidade. Tais valores foram calculados, em cada janela de dados considerada, ao agrupar as estimativas de mesma latência. Exemplificando 0 caso apresentado na Figura 2 (janela de 6 horas), os valores do viés e do EMQ foram calculados considerando as 720 estimativas geradas para cada intervalo de 5 minutos de latência dos 360 que compõem tal janela. 0 número de pares de dados efetivamente utilizados foi diferente deste número total possível, pois houve falhas nas estimativas do ZTD em algumas épocas dentro do período considerado, devido a problemas nos dados. 0 percentual de dados utilizados nessa análise foi em média de 94,6\%. Isso representa um número médio de 680 pares de dados considerados e mostra que os resultados aqui apresentados são estatisticamente representativos. A Figura 3 apresenta os valores dos indicadores de qualidade do IWV-GPS para as diferentes janelas avaliadas.

Os valores negativos do viés apresentados na Figura 3 mostram que as estimativas em tempo real apresentam uma tendência de serem menores do que as estimativas pós-processadas, utilizadas aqui como referência. Monico et al. (2001) e Sapucci et al. (2007), mostraram que as estimativas pós-processadas apresentam uma tendência de superestimar os valores do IWV 

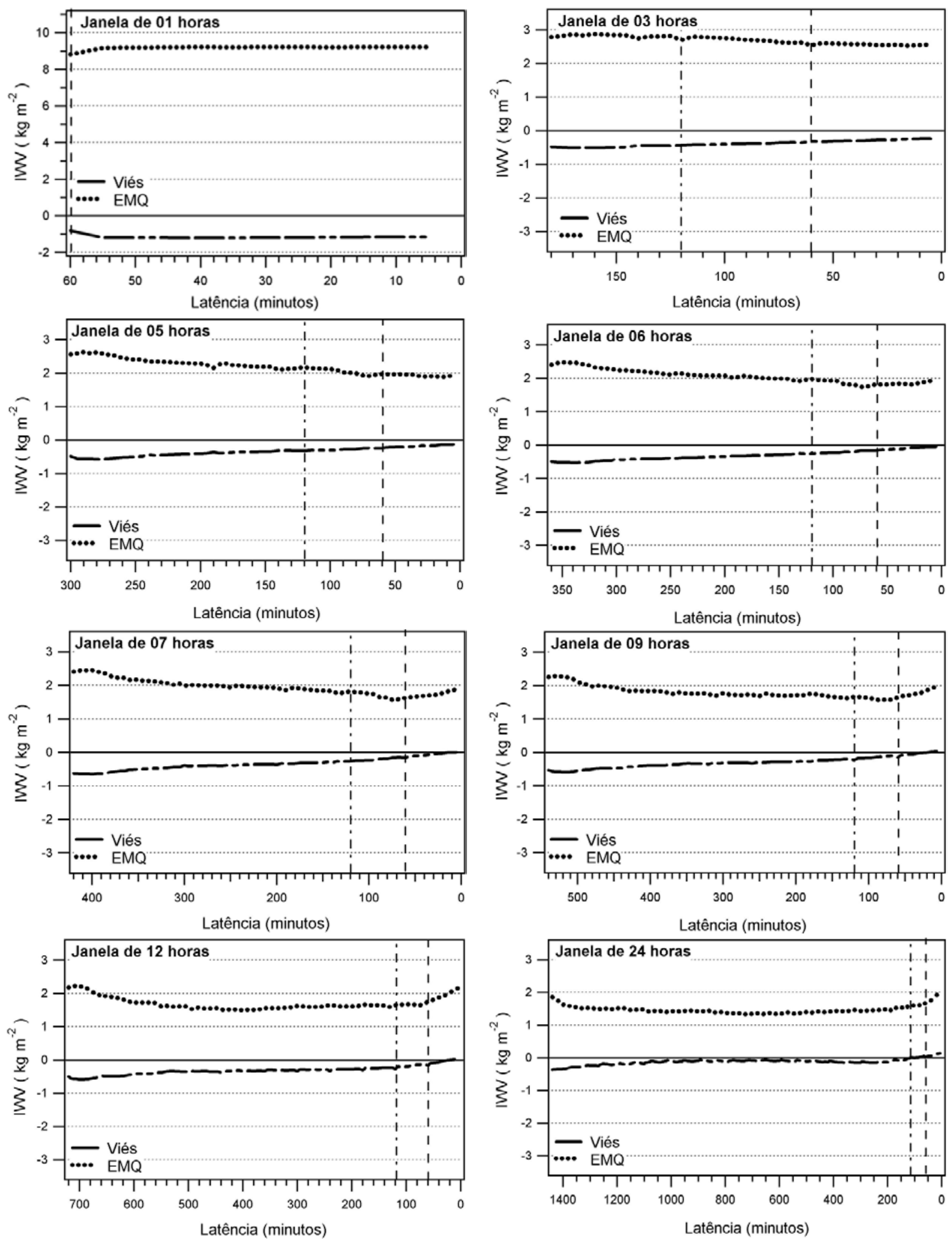

Figura 3 - Indicadores da qualidade dos valores do IWV-GPS em função da latência para diferentes janelas deslizantes de dados. Os traços na vertical sinalizam a latência de 60 minutos (tracejado) e 120 minutos (traço e ponto). 
com relação às radiossondas. Ao ponderar os valores do viés apresentados aqui com esses resultados verifica-se que no processamento em tempo real essa tendência com relação às radiossondas é amenizada. A provável causa desse fato pode ser a combinação dos erros presentes nesses processos (como a incerteza nas efemérides e nas correções dos relógios dos satélites bem como os efeitos de segunda ordem da lonosfera, entre outros) que, apesar de também estarem presentes no pós-processado, se compensam gerando um saldo positivo à diminuição das estimativas do IWV-GPS em tempo real.

Os resultados mostrados na Figura 3 indicam que a janela de 1 hora apresenta valores do viés e EMQ com um padrão diferente das demais janelas avaliadas. Enquanto que com a janela de 1 hora os valores do viés e do EMQ independem da latência, permanecendo constantes, as demais janelas avaliadas apresentam valores do viés suavemente crescente com a latência, e valores do EMQ oscilantes dentro do intervalo que compõem cada janela. Outro ponto que diferencia a janela de 1 hora das demais está relacionado com a magnitude de tais estatísticas. Com a janela de 1 hora os valores do EMQ e viés foram praticamente constantes (9,0 $\mathrm{kg} \mathrm{m}^{-2}$ e $-1,0 \mathrm{~kg} \mathrm{~m}^{-2}$, respectivamente). Considerando outras janelas, a qualidade das estimativas melhora significativamente, pois o EMQ é sempre menor que $3,0 \mathrm{~kg} \mathrm{~m}^{-2}$ e 0 viés é maior que $-0,8 \mathrm{~kg} \mathrm{~m}^{-2}$ (observe a modificação na escala vertical da Fig. 3a).

Considerando os valores do $E M Q$ e excluindo dessa análise a janela de 1 hora, as janelas podem ser divididas em três classes: uma primeira composta pela janela de 3 horas, a qual apresentou valores do EMQ acima de $2,5 \mathrm{~kg} \mathrm{~m}^{-2}$; uma segunda composta pelas janelas de 5, 6, 7, 9 e 12 horas de dados, as quais apresentaram valores do EMQ entre 1,5 a 2,5 $\mathrm{kg} \mathrm{m}^{-2}$; e uma terceira composta por apenas a janela de 24 horas, a qual apresentou valores menores que $2,0 \mathrm{~kg} \mathrm{~m}^{-2}$ independente da latência. As janelas que compõem tais classes são caracterizadas também por apresentarem valores do EMQ maiores nas extremidades da janela do que nas épocas intermediárias. Esse comportamento dos valores do EMQ é mais explícito quanto maior o período considerado, pois na janela de 3 horas ele é quase imperceptível, enquanto que nas janelas de 12 e 24 horas torna-se evidente. Como discutido na segunda seção, a causa desse comportamento são os processos de suavização e filtragem que não são adequadamente executados em todas as épocas da janela. Em conseqüência desse comportamento, verifica-se que há um pico de mínimo EMQ em todas as janelas avaliadas, o qual está associado a uma determinada latência. Tais informações são aqui utilizadas para nortear a escolha da janela de dados que otimiza a obtenção das estimativas do IWV-GPS. Na Tabela 1 são apresentados quantitativamente a latência e demais indicadores de qualidade para os picos de mínimo EMQ das diferentes janelas consideradas, resumindo os melhores resultados apresentados na Figura 3.

Um aspecto importante que pode ser destacado nos resultados apresentados na Tabela 1 é que, para aplicações onde a latência das estimativas do IWV é uma característica mais relevante que a qualidade, a melhor opção é fornecida com a utilização de uma janela de dados de 3 horas, pois valores com um EMQ de 2,5 $\mathrm{kg} \mathrm{m}^{-2}$ são obtidos com uma latência de apenas 15 minutos. Por outro lado, os valores apresentados na Tabela 1 sugerem que estimativas do IWV-GPS de melhor qualidade (EMQ de 1,33 $\mathrm{kg} \mathrm{m}^{-2}$ ) são obtidas utilizando uma janela de dados de 24 horas. Esse fato justifica a escolha dessa janela em procedimentos onde o tempo de processamento não é um fator crítico. Porém, a latência necessária para se obter tal qualidade (730 minutos) limita a utilização dessas estimativas em diversas aplicações operacionais como, por exemplo, a assimilação de dados. 0 mesmo ocorre com a utilização de janelas de 12 horas, pois a latência necessária para se obter valores de melhor qualidade (EMQ de 1,48 $\mathrm{kg} \mathrm{m}^{-2}$ ) é de 400 minutos. Os valores da latência necessária para se obter a melhor qualidade utilizando as demais janelas não apresentam esse problema, pois são iguais ou menores que 90 minutos. Como há uma diminuição da qualidade das estimativas do IWV-GPS ao empregar essas janelas, na definição da janela mais apropriada para a assimilação operacional de dados é necessário avaliar outros aspectos envolvidos.

\section{Definição da janela de dados que otimiza operacionalmente a obtenção do IWV-GPS}

Como a obtenção das estimativas IWV-GPS requer um processo que converte as estimativas do ZTD em valores do IWV, do ponto de vista operacional, a latência das estimativas geradas pelo processamento GPS deve ser menor que a latência máxima permitida pelo sistema de assimilação. Considerando também que a coleta dos dados GPS tem sido feita em blocos de 1 hora, intervalos de alguns minutos, como foi a latência (1 hora e 15 minutos) do melhor resultado obtido com a janela de 7 horas, não é viável para fins operacionais. Logo, a latência para a estimativa do ZTD não deve ser maior que 60 minutos, destinadas para aplicações de nowcast (em uma configuração futura), e de 120 minutos, destinada para a atual configuração operacional do CPTEC. Para evidenciar esse problema, na Figura 3 foram colocados traços indicadores da latência de 60 e 120 minutos, 
Tabela 1 - Latência dos valores do IWV-GPS de melhor qualidade para as diferentes janelas deslizantes de dados consideradas.

\begin{tabular}{|c|c|c|c|c|c|}
\hline $\begin{array}{c}\text { Janela de } \\
\text { dados }\end{array}$ & $\begin{array}{c}\text { Número de dados } \\
\text { considerados }\end{array}$ & $\begin{array}{c}\text { Latência } \\
\text { (minutos) }\end{array}$ & $\begin{array}{c}\text { Viés } \\
\left(\mathrm{kg} \mathrm{m}^{-2}\right)\end{array}$ & $\begin{array}{c}\text { Desvio padrão } \\
\left(\mathrm{kg} \mathrm{m}^{-2}\right)\end{array}$ & $\begin{array}{c}\text { EMQ } \\
\left(\mathrm{kg} \mathrm{m}^{-2}\right)\end{array}$ \\
\hline 01 hora & 650 & 60 & $-0,80$ & 8,78 & 8,81 \\
\hline 03 horas & 674 & 15 & $-0,24$ & 2,52 & 2,53 \\
\hline 05 horas & 649 & 30 & $-0,17$ & 1,88 & 1,88 \\
\hline 06 horas & 653 & 75 & $-0,17$ & 1,74 & 1,75 \\
\hline 07 horas & 646 & 75 & $-0,15$ & 1,55 & 1,56 \\
\hline 09 horas & 647 & 90 & $-0,14$ & 1,57 & 1,58 \\
\hline 12 horas & 659 & 400 & $-0,32$ & 1,45 & 1,48 \\
\hline 24 horas & 635 & 730 & $-0,10$ & 1,33 & 1,33 \\
\hline
\end{tabular}

os quais mostram que os melhores resultados normalmente não coincidem com as latências operacionalmente úteis. No entanto, é com essas latências que se deve avaliar qual é a configuração mais adequada para a otimização do processamento dos dados GPS. A Figura 4 apresenta os valores do EMQ obtidos com a utilização das janelas de dados avaliadas fixando-se a latência em 60 (Fig. 4a) e 120 minutos (Fig. 4b).

Ao considerar apenas a qualidade obtida das estimativas, observa-se na Figura 4 que para a latência de 60 minutos a janela de 7 horas é a mais adequada, enquanto que para a latência de 120 minutos a janela de 24 horas é mais apropriada. No entanto, nesse estudo outro aspecto deve ser considerado, que é o custo computacional. Ou seja, o tempo ( $t$ ) gasto pelo computador para o processamento dos dados utilizando as diferentes janelas de dados. Na mesma Figura 4 é apresentado também o tempo médio de máquina com as diferentes janelas de dados avaliadas aqui. Observa-se que a partir da janela de 7 horas de dados 0 tempo de processamento cresce mais acentuadamente, enquanto que nesse mesmo limiar a qualidade final das estimativas não tem um ganho significativo. Para definir qual é o tamanho da janela de dados mais adequado para otimizar o processamento dos dados GPS, deve-se considerar simultaneamente essas duas características. Para isso é proposta uma metodologia baseada na distância euclidiana $(D)$ de uma janela (win) composta de coordenadas $\left(E M Q_{\text {win }}, t_{\text {win }}\right)$ à origem $(0,0)$ em um espaço bidimensional, composto por valores do EMQ e de t normalizados pelos valores máximos da amostra em estudo. Os valores de $D_{W I N}$ são obtidos aplicando a seguinte equação:

$$
D_{W I N}=\sqrt{\left(\frac{E M Q_{W I N}}{E M Q_{M A X}} \cdot P_{E M Q}\right)^{2}+\left(\frac{T_{W I N}}{t_{M A X}} \cdot P_{t}\right)^{2}},
$$

onde os valores de $E M Q_{M A X}$ e $t_{M A X}$ utilizados foram
8,81 $\mathrm{kg} \mathrm{m}^{-2}$ (janela de 1 hora) e 43,58 s (janela de 24 horas), respectivamente. Os valores de $P_{E M Q}$ e $P_{t}$ são os pesos dos valores do EMQ e do $t$. Nessa análise, foi considerado que a importância dos valores do EMQ teria que ser duas vezes maior que a importância de $t$, para que 0 ganho em tempo de processamento não prejudique a qualidade final das estimativas do IWV-GPS. Dessa forma os valores de $P_{E M Q}$ e $P_{t}$ aplicados na Eq. (4) foram 2 e 1 , respectivamente. Os valores da Eq. (4) para as diferentes janelas de dados avaliadas são apresentados na Figura 5. Para se chegar na janela de dados que otimiza o processamento (W I $N_{\text {otima }}$ ) basta minimizar a Eq. (4), ou seja:

$$
\begin{aligned}
W I N_{\text {OTIMA }}= & \operatorname{Min}\left(D_{W I N}\right), \\
& W I N=1,3,5,6,7,9,12,24 .
\end{aligned}
$$

Observando os valores apresentados na Figura 5 nota-se que 0 mínimo da Eq. (4) é obtido ao utilizar uma janela de 7 horas (valor em destaque nessa figura). Com essa análise pode-se afirmar que a utilização de uma janela de dados de 7 horas fornece a melhor relação entre tempo de processamento e qualidade final das estimativas do IWV-GPS, tanto para a latência de 60 como de 120 minutos.

\section{CONCLUSÕES}

0 presente trabalho apresentou uma avaliação do tamanho adequado da janela de dados a ser utilizada para se obter a meIhor combinação entre latência, tempo de processamento e qualidade final das estimativas do IWV-GPS. Janelas de 1, 3, 5, 6, 7, 9,12 e 24 horas de dados foram testadas em simulações de processamento em tempo real ao empregar efemérides ultra-rápidas e dados GPS coletados durante o mês de junho de 2004. A qualidade das estimativas foi avaliada ao compará-las com as provenientes do pós-processamento dos mesmos dados utilizando 


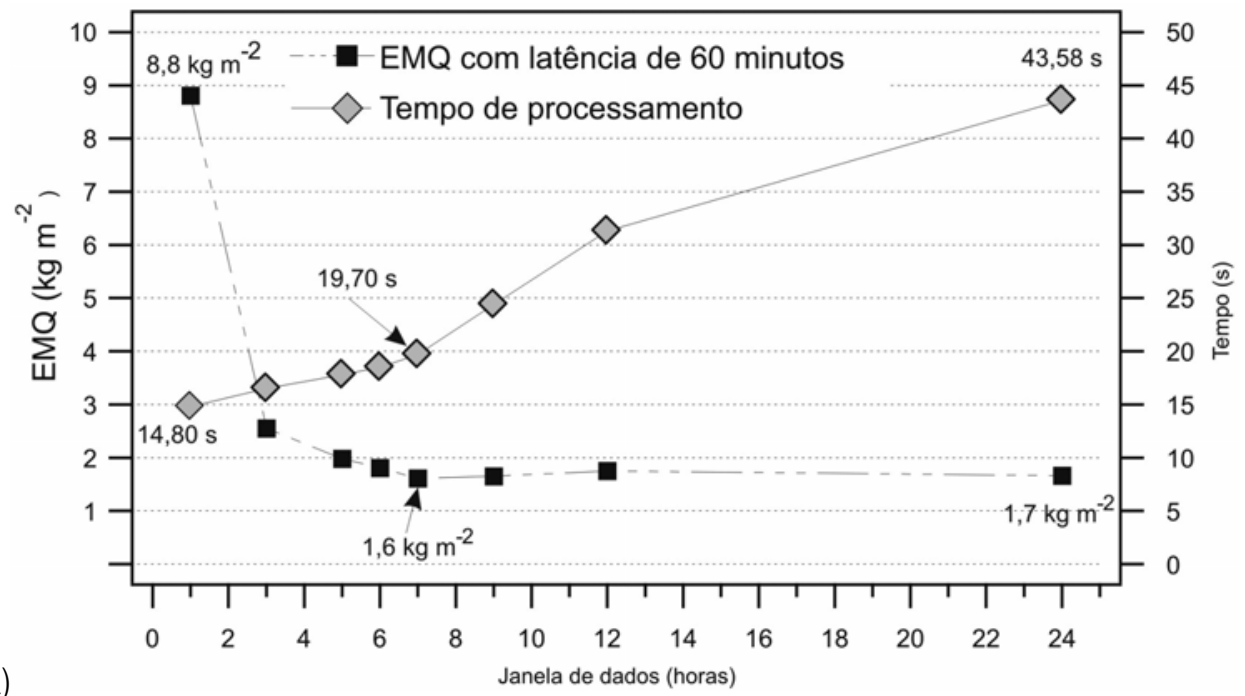

(a)

Janela de dados (horas)

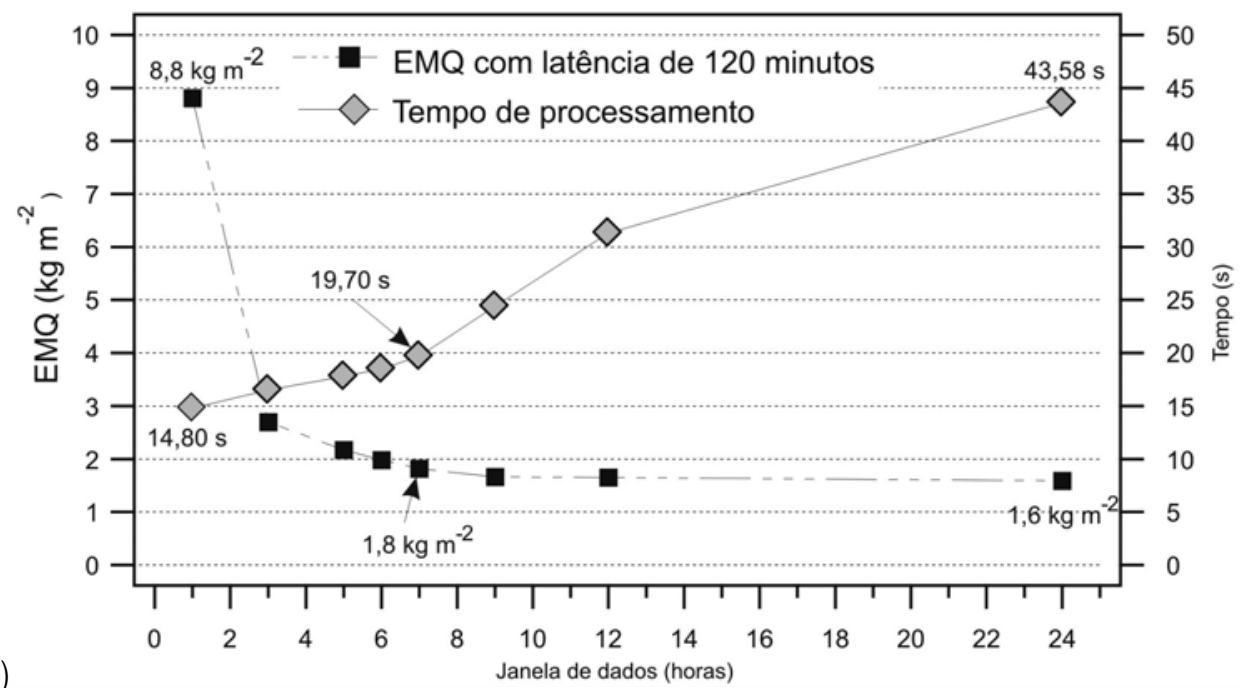

(b)

Figura 4 - Valores do EMQ (tracejado) obtidos com uma latência fixa de (a) 60 minutos e (b) 120 minutos e 0 tempo de processamento (traço contínuo), ambos em função do tamanho das janelas de dados.

efemérides precisas. Os resultados obtidos permitiram concluir que:

- Para aplicações onde a latência dos dados é uma característica mais relevante que a qualidade, a janela de 3 horas de dados é a mais adequada, pois fornece valores do IWV com EMQ de 2,5 $\mathrm{kg} \mathrm{m}^{-2}$ com uma latência de apenas 15 minutos;

- Para aplicações onde 0 tempo de processamento e a latência não são fatores críticos a janela de dados de 24 horas é a mais apropriada, pois fornece a melhor qualidade (EMQ de 1,33 $\mathrm{kg} \mathrm{m}^{-2}$ ) entre as janelas avaliadas;
- Para aplicações na assimilação de dados, tanto para previsão de nowcast (latência permitida de 60 minutos) quanto que para previsões de médio e longo prazo (latência permitida de 120 minutos), a janela de 7 horas é a que forneceu os melhores resultados ao considerar simultaneamente 0 tempo de processamento e a qualidade final das estimativas IWV-GPS.

Os resultados obtidos na definição da janela de dados que otimiza o processamento GPS agrega uma significativa contribuição à assimilação de dados IWV-GPS, dado o crescente número de estações GPS disponíveis para essa aplicação no Brasil. Outro 


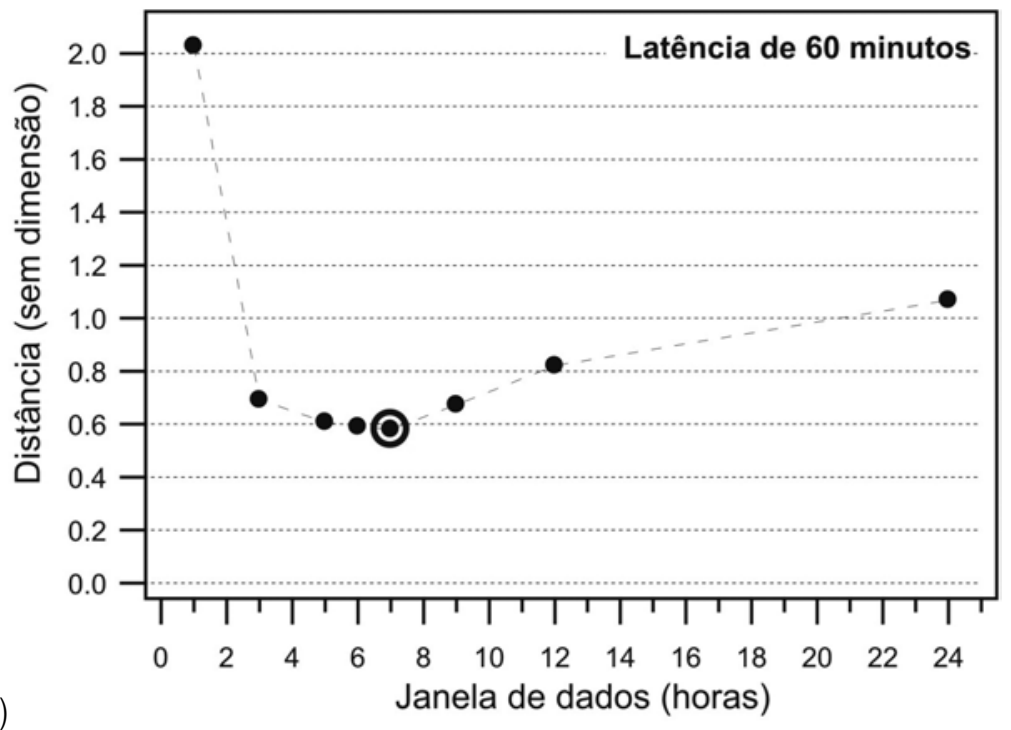

(a)

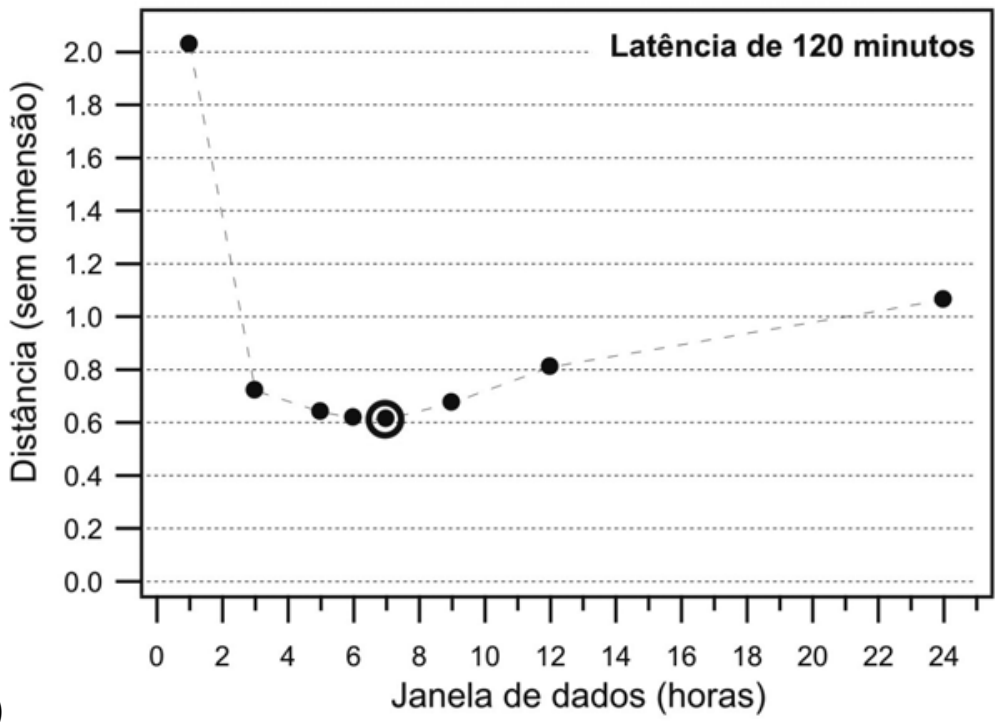

Figura 5 - Distância euclidiana gerada pela Eq. (4) em função do tamanho das janelas de dados para a latência de (a) 60 e (b) 120 minutos.

aspecto relacionado ao tema que deve ser destacado é a aplicação do IWV-GPS para a previsão de fortes tempestades que tem sido recentemente investigada. Nessa aplicação a latência e a qualidade dos dados são muito importantes. Os resultados aqui apresentados viabilizam não apenas a utilização de diversas estações GPS, mas também a diminuição dos intervalos de tempo entre os processamentos (que é de 1 hora, para 15 minutos, ou até mesmo 5 minutos) aumentando o desempenho dessa aplicação GPS. Como o experimento realizado considerou apenas dados coletados durante um único mês (na transição entre outono e inverno), outros experimentos contendo dados coletados durante outras estações do ano devem ser realizados para avaliar o impacto da sazonalidade do IWV nos resultados apresentados.

\section{AGRADECIMENTOS}

Os autores agradecem: ao JPL (Jet Propulsion Laboratory), pela disponibilidade das efemérides ultra-rápidas e precisas utilizadas no processamento dos dados; à FAPESP - Fundação de Amparo à Pesquisa no Estado de São Paulo (proc. n 01/12761-9), pelo suporte financeiro durante o período em que os experimentos foram feitos. 


\section{REFERÊNCIAS}

BEVIS M, BUSINGER S, HERRING TA, ROCKEN C, ANTHES RA \& WARE RH. 1992. GPS Meteorology: Remote of Atmospheric Water Vapor Using the Global Positioning System. J. Geophys. Res., 97: 15787-15801.

BEVIS M, CHISWELL G, HERRING TA, ANTHES R, ROCKEN C \& WARE RH. 1994. GPS Meteorology: mapping zenith wet delays into precipitable water. J. Appl. Meteor., 33: 379-386.

BLACK TL. 1994. The New NMC mesoscale Eta model: description and forecast examples. Wea. Forecasting, 9: 256-278.

BLEWITT G. 1997. Advances in Global Positioning System Technology for Geodynamics Investigations: 1978-1992 in An Introduction to GIPSY/OASIS-II. JPL 4800 Oak Grove Drive, Pasadena, CA 91109. $288 \mathrm{pp}$.

CAVALCANTI IFA, MARENGO JA, SATYAMURTY P, NOBRE CA, TROSNIKOV I, BONATTI JP, MANZI AO, TARASOVA T, PEZZI LP, D'ALMEIDA C, SAMPAIO G, CASTRO CC, SANCHES MB \& CAMARGO H. 2002. Global climatological features in a simulation using CPTEC/COLA AGCM. J Climate, 15: 2965-2988.

COHN SE, DA SILVA A, GUO J, SIENKIEWICZ M \& LAMICH D. 1998. Assessing the effects of data selection with the DAO Physical-space Statistical Analysis System. Mon. Wea. Rev., 126: 2913-2926.

DAVIS JL, HERRING TA, SHAPIRO I, ROGERS AE \& ELGENED G. 1985. Geodesy by Interferometry: Effects of Atmospheric Modeling Errors on Estimates of Base Line Length. Radio Sci., 20: 1593-1607.

DA SILVA A \& GUO DJ. 1996. Documentation of the Physical-space Statistical Analysis System (PSAS) Part I: The Conjugate Gradient Solver Version PSAS-1.00. DAO Office Technical Note 96-02, pp. 6. 1996. Disponível em: < http://dao.gsfc.nasa.gov/subpages/office-notes.html> Acesso em: 12 set. 2005.

FORTES LPS. 1997. Operacionalização da Rede Brasileira de Monitoramento Contínuo do Sistema GPS (RBMC). Rio de Janeiro, (Dissertação de Mestrado - Computação do Instituto Militar de Engenharia). 152 p.

FOSTER J, BEVIS M \& BUSINGER S. 2005. GPS Meteorology: Slidingwindow analysis J. Atmos. Oceanic Technol., 22: 687-695.

GREGORIUS T. 1996. How it Works... GIPSY OASIS II, Department of Geomaties University of Newcastle upon Tyne. Internal Publication. 167 pp.

HERDIES DL, FERREIRA SHS, CINTRA R \& BONATTI JP. 2002. 0 sistema de assimilação de dados atmosféricos global do CPTEC/INPE. In: Anais do XII Congresso Brasileiro de Meteorologia, Foz do Iguaçu - PR. CD-ROM.

KALNAY E. 2003. Atmospheric Modeling, Data Assimilation and Predictability. Cambridge University Press, London, 341 pp.

KINTER JL, DEWITT DG, DIRMEYER PA, FENNESSY MJ, KIRTMAN BP, MARX L, SCHNEIDER EK, SHUKLA J \& STRAUS D. 1997. The COLA
Atmosphere-Biosphere General Circulation Model Volume 1: Formulation. COLA Tech. Rep. 51, 46 pp. [Available from COLA 4041 Powder Mill Road, Suite 302, Calverton, MD 20705.]

KLOBUCHAR JA. 1996. Ionospheric Effects on GPS. In: PARKINSON BW \& SPILKER Jr JJ (Ed.). Global Positioning System: Theory and Applications. American Institute of Aeronautics and Astronautics, 1: 485-515.

KUO YH, ZUO X \& GUO YR. 1996. Variational Assimilation of Precipitable Water Using Nonhydrostatic Mesoscale Adjoin Model. Part I: Moisture retrieval and sensitivity experiments. Mon. Wea. Rev., 124: 122-147.

MAREL H. 2000. Exploitation of ground based GPS for climate and numerical weather prediction application Status of Cost action 716. Proc. of the EUREF Symposium, 1999, Prague, Czech Republik, 60, 51-53, Bayerischen Akademie der Wissenschaften.

MONICO JFG. 2000. Posicionamento por Ponto de Alta Precisão Utilizando 0 GPS: Uma Solução para a Geodinâmica. Rev. Bras. Geof., 18 39-48.

MONICO JFG, SAPUCCI LF \& TOMMASELLI JTG. 2001. 0 GPS no Suporte à Meteorologia: Perspectivas de Aplicações no Brasil. Bol. Soc. Bras. Met., 25: 7-15.

NASA. National Aeronautics Space Administration. 2005. IGS International GNSS Service. Disponível em: < http://igscb.jpl.nasa.gov> Acesso em: 12 set. 2005.

NIELL AE. 1996. Global Mapping Functions for the Atmosphere Delay at Radio Wavelengths. J. Geophys. Res., 101: 3227-3246.

REIGBER C, GENDT G, DICK G \& TOMASSINI M. 2001. Near real-time water vapor monitoring in a German GPS network and assimilation into weather forecast model. In: $14^{\text {th }}$ IONGPS International Meeting of Institute of Navigation: 2001, Utah. Proceedings of the IONGPS International Meeting of Institute of Navigation. Utah. 2001. CD-ROM.

ROCKEN C, VAN HOVE T \& WARE RH. 1997a. Near Real-Time GPS Sensing of Atmospheric Water Vapor. Geophys. Res. Lett., 24: 3221-3224.

ROCKEN C, ANTHES R, EXNER M, HUNT D, SOKOLOVSKIY S, WARE R, GORBUNOV M, SCHREINER W, FENG D, HERMAN B, KUO Y \& ZOU X. 1997b. Analysis and validation of GPS/MET data in the neutral atmosphere. J. Geophys. Res., 102: 29849-29866.

SAPUCCI LF. 2001. Precipitable Water Measurements Using GPS: a Case Study in Brazil. In: $14^{\text {th }}$ Inter. Tec. Meeting of Institute of Navigation, 14: 2001, Utah. Proceedings of the IONGPS $14^{\text {th }}$ International Technical Meeting of the Satellite Division of the Institute of Navigation. Utah. 2001. CD-ROM.

SAPUCCI LF, MACHADO LAT \& MONICO JFG. 2004. Modelagem da temperatura média troposférica no Brasil para quantificação do IWV utilizando GPS. In: Congr. Bras. Met., 13.: 2004, Fortaleza. Anais do XIII Congresso Brasileiro de Meteorologia. Fortaleza: SBMET, 2004. CDROM 
SAPUCCI LF, MACHADO LAT, MONICO JFG \& PLANA-FATORI A. 2007. Intercomparison of Integrated Water Vapor Estimative from Multi-sensor in Amazonian Regions. J. Atmos. Oceanic Technol., 24: 1880-1894.

SPILKER Jr JJ. 1996. Tropospheric Effects on GPS. In: PARKINSON BW \& SPILKER Jr JJ (Ed.). Global Positioning System: Theory and Applications. American Institute of Aeronautics and Astronautics, p. 517-546.

TSUDA T, HEKI K, MIYAZAKI S, AONASHI K, HIRAHARA K, NAKAMURA
H, TOBITA M, KIMATA F, TABEI T, MATSUSHIMA T, KIMURA F, SATOMURA M, KATO T \& NAITO I. 1998. GPS meteorology project of Japan - exploring frontiers of geodesy. Earth Planets Space, 50: 1-5.

WARE R, FULKER DW, STEIN SA, ANDERSON DN, AVERY SK, CLARK RD, DROEGEMEIER KK, KUETTNER JP, MINSTER JB \& SOROOSHIAN S. 2000. SUOMINET: A Real-Time National GPS Network for Atmospheric Research and Education. Bull. Amer. Meteor. Soc., 81: 677-694.

\section{NOTAS SOBRE OS AUTORES}

Luiz Fernando Sapucci. Matemático graduado em 1998 pela FCT-UNESP de Presidente Prudente. Recebeu os títulos de Mestre e Doutor em Ciências Cartográficas em 2001 e 2005, respectivamente, pela mesma instituição ao investigar a potencialidade do IWV-GPS à meteorologia no Brasil. Atualmente atua no Grupo de Assimilação de Dados da Divisão de Desenvolvimento e Modelagem do CPTEC, sendo responsável pela assimilação e avaliação de medidas de umidade provenientes de fontes não convencionais, como satélites e sensores em bases terrestres.

João Francisco Galera Monico. Engenheiro Cartógrafo pela FCT/UNESP - Campus de Presidente Prudente, com Doutorado em Engenharia de Levantamentos e Geodésia Espacial pela University of Nottingham - UK. Atualmente é Professor Livre Docente do Departamento de Cartografia da FCT/UNESP onde desenvolve pesquisas relacionadas com GNSS e leciona na graduação em Engenharia Cartográfica e no programa de pós-graduação em Ciências Cartográficas. É pesquisador do CNPq nível 1-C.

Luiz Augusto Toledo Machado. Possui Bacharelado em Meteorologia pela Universidade de São Paulo (1981), mestrado em Meteorologia pela Universidade de São Paulo (1984), Diplôme d’Études Approfondies Oceanologie Meteorologie - Université de Paris VI (Pierre et Marie Curie) (1989), e doutorado em Sciences de La Vie - Université de Paris VI (Pierre et Marie Curie) (1992). Foi pesquisador visitante da NASA/GISS e do LMD/França. Membro do corpo Docente da PGMET do INPE. Atualmente é pesquisador titular III do Instituto Nacional de Pesquisas Espaciais e chefe da Divisão de Satélites e Sistemas Ambientais do CPTEC/INPE.

Dirceu Luis Herdies. Possui graduação em Física pela Universidade Federal de Santa Maria (1983), mestrado em Meteorologia pelo Instituto Nacional de Pesquisas Espaciais - INPE (1991), doutorado em Meteorologia pela Universidade de São Paulo (2002) e concluiu o pós-doutorado no NCEP/NOAA nos Estados Unidos em 2006. Atualmente é pesquisador associado do CPTEC/INPE, sendo coordenador do Grupo de Assimilação de Dados da Divisão de Modelagem e Desenvolvimento. Foi pesquisador visitante da GSFC/NASA em 2000 e 2001 e da Universidade de Maryland e NCEP/NOAA em 2005.

Rodrigo Augusto Ferreira de Souza. Possui graduação em Física pela Universidade Estadual Paulista - UNESP (1995), mestrado em Meteorologia pelo Instituto Nacional de Pesquisas Espaciais - INPE (1999), doutorado em Meteorologia pelo Instituto Nacional de Pesquisas Espaciais - INPE (2004). Atualmente, é pesquisador da Divisão de Satélites e Sistemas Ambientais do Centro de Previsão de Tempo e Estudos Climáticos (DSA/CPTEC/INPE) e responsável pela área de sondagem remota da atmosfera. 